\title{
Discordant Risk: Overweight and cardiometabolic risk in Chinese adults
}

\author{
Penny Gordon-Larsen, PhDa,b, Linda S. Adair, PhD ${ }^{a, b}$, James B. Meigs, MD $^{c}$, Elizabeth \\ Mayer-Davis, $\mathbf{P h D}^{\mathrm{a}, \mathrm{d}}$, Amy Herring, $\mathbf{P h D}^{\mathrm{c}, \mathrm{e}}$, Shengkai Yan, $\mathbf{M D}^{f}$, Bing Zhang, MD, $\mathbf{P h D}^{\mathrm{g}}$, Du \\ Shufa, MD, PhD ${ }^{a, b}$, and Barry M. Popkin, PhD ${ }^{a, b}$ \\ aDepartment of Nutrition, Gillings School of Global Public Health at the University of North \\ Carolina \\ ${ }^{b}$ Carolina Population Center, University of North Carolina \\ ${ }^{d}$ General Medicine Division, Massachusetts General Hospital and Harvard Medical School \\ ${ }^{\mathrm{d}}$ Department of Medicine, University of North Carolina \\ eDepartment of Biostatistics, Gillings School of Global Public Health at the University of North \\ Carolina \\ ${ }^{f}$ China-Japan Friendship Hospital \\ gNational Institute of Nutrition and Food Safety, China Center for Disease Control, Beijing China
}

\section{Abstract}

Recent US work identifies "metabolically healthy overweight" and "metabolically at risk normal weight" individuals. Less is known for modernizing countries with recent increased obesity. Fasting blood samples, anthropometry and blood pressure from 8,233 adults aged 18-98 in the 2009 nationwide China Health and Nutrition Survey, were used to determine prevalence of overweight (Asian cut point, BMI $\geq 23 \mathrm{~kg} / \mathrm{m}^{2}$ ) and five risk factors [pre-diabetes/diabetes (HbA1c $25.7 \%$ ) inflammation (hsCRP $\geq 3 \mathrm{mg} / \mathrm{L}$ ), pre-hypertension/hypertension (SBP/ DBP $\geq 130 / 85 \mathrm{mmHg}$ ), high triglycerides ( $\geq 150 \mathrm{mg} / \mathrm{dL}$ ), low high-density lipoprotein cholesterol $(<40$ (men) $/<50 \mathrm{mg} / \mathrm{dL}$ (women)]. Sex-stratified, logistic and multinomial logistic regression models estimated concurrent obesity and cardiometabolic risk, with and without abdominal obesity, adjusting for age, smoking, alcohol consumption, physical activity, urbanicity, and income. Irrespective of urbanicity, $78.3 \%$ of the sample had $\geq 1$ elevated cardiometabolic risk factor (normal weight: $33.2 \%$ had $\geq 1$ elevated risk factor; overweight: $5.7 \%$ had none). At age 18 $30,47.4 \%$ had no elevated risk factors, which dropped to $6 \%$ by age 70 , largely due to age-related increase in hypertension risk (18-30 years: 11\%, >70 years: $73 \%$ ). Abdominal obesity was highly

Users may view, print, copy, and download text and data-mine the content in such documents, for the purposes of academic research, subject always to the full Conditions of use:http://www.nature.com/authors/editorial_policies/license.html\#terms

Please Address Correspondence and Reprint Requests To: Penny Gordon-Larsen, Ph.D., University of North Carolina at Chapel Hill, Carolina Population Center (University Square), 123 West Franklin Street, Chapel Hill, NC 27516-3997, Phone: (W) 919-843-9966; Fax: 919-966-9156, pglarsen@unc.edu.

Conflict of Interest

None. The authors declare no conflict of interest. None of the individuals acknowledged received compensation for any role in the study. 
predictive of metabolic risk, irrespective of overweight (e.g., "metabolically at risk overweight" relative to "metabolically healthy normal weight" [men: Relative Risk Ratio (RRR) $=39.06$; 95\% Confidence Interval (CI): 23.47, 65.00; women: RRR=22.26; 95\% CI: 17.49, 28.33]). To conclude, a large proportion of Chinese adults have metabolic abnormalities. High hypertension risk with age, irrespective of obesity underlies the low prevalence of metabolically healthy overweight. Screening for cardiometabolic-related outcomes dependent upon overweight will likely miss a large portion of the Chinese at-risk population.

\section{INTRODUCTION}

In the US, approximately 16.3 million adults are of normal weight but have two or more metabolic abnormalities.(1) Understanding heterogeneity in risk by overweight status is important particularly in the metabolically at risk but non-overweight population as these individuals often elude screening as they are not perceived as high risk. However, little is known about heterogeneity in risk in Asian populations, and particularly in China where rates of obesity-related non-communicable diseases have recently increased and are now the leading causes of morbidity, disability, and mortality. $(2,3)$

Given that Asians are known to have relatively high abdominal obesity(4) and high prevalence of cardiometabolic risk factors at relatively low BMI(5) and at younger ages,(6) understanding how abdominal obesity associates with cardiometabolic risk in the absence of (and in combination with) obesity is important. Although abdominal obesity does not confer the same level of risk for hypertension, diabetes, inflammation and dyslipidemia, many studies rely on syndrome-based indicators (e.g., metabolic syndrome(7, 8), Framingham risk score $(9,10))$ and thus may miss important variation that can be observed by investigating variation in co-occurrence of obesity and cardiometabolic risk across individual risk factors.

Understanding the distribution of cardiometabolic risk by age and across overweight and non-overweight individuals is essential for determining the impending burden of cardiovascular disease in China, home to more than 1.3 billion people and comprising one fifth of the world's population. The distribution of cardiometabolic risk factors, particularly among the non-overweight population is particularly important for prevention as these individuals are likely to be missed in screening efforts. To this end, a national Chinese cohort was examined to determine the prevalence of "metabolically healthy overweight" and "metabolically at risk normal weight" by age. Analyses were structured to test the hypothesis that the association between overweight and five risk factors [pre-diabetes/ diabetes (HbA1c $\geq 5.7 \%$ ) inflammation (hsCRP $\geq 3 \mathrm{mg} / \mathrm{L}$ ), pre-hypertension/hypertension (SBP/DBP $\geq 130 / 85 \mathrm{mmHg}$ ), high triglycerides ( $\geq 150 \mathrm{mg} / \mathrm{dL}$ ), low high-density lipoprotein cholesterol ( $<40$ (men)/ $<50 \mathrm{mg} / \mathrm{dL}$ (women)] varies, depending upon the indicator. Further analyses tested independent and interactive effects of overweight and abdominal obesity across pre-hypertension or hypertension, pre-diabetes or diabetes, high triglycerides, low HDL-C, and inflammation. 


\section{MATERIALS \& METHODS}

\section{The China Health and Nutrition Survey (CHNS)}

The CHNS collected health data in 228 communities [nine diverse provinces (Guangxi, Guizhou, Heilongjiang, Henan, Hubei, Hunan, Jiangsu, Liaoning, and Shandong)] throughout China from 1989-2009 with eight rounds of surveys, with the 2009 survey collecting fasting blood for the first time. Using multistage, random cluster sampling, counties in the nine provinces were stratified by income and weighted sampling was used to randomly select four counties in each province. Villages and small towns within counties and urban and suburban neighborhoods within cities were selected randomly into primary sampling units that were politically and geographically classified based on State Statistical Office definitions. The surveyed provinces represent $56 \%$ of the Chinese population. Survey procedures have been described elsewhere.(11) The study was approved by the Institutional Review Board at the University of North Carolina at Chapel Hill, the China-Japan Friendship Hospital, Ministry of Health and China, and Institute of Nutrition and Food Safety, China Centers for Disease Control and subjects gave informed consent for participation.

\section{Analysis Sample}

A total of 10,038 adult respondents were surveyed at the 2009 exam, 1,423 did not give blood, 24 were not fasting, and 62 were pregnant, resulting in a total of 8,529 individuals with fasting blood samples. Of these, 237 were missing anthropometry and 60 were missing lab results, resulting in an analytic sample of 8,233 with anthropometry and clinical exam data. There were no statistically significant differences in the total 2009 sample versus the analytic sample in income, urbanization, BMI, cardiometabolic risk or overweight, although the analytic sample included more women, was slightly younger, and had slightly larger waist circumference.

\section{Measures}

Cardiometabolic Risk-Following an overnight fast, blood was collected by venipuncture (12-ml). Whole blood was immediately centrifuged and serum glucose testing completed. The remaining plasma and serum samples were immediately frozen and stored for later laboratory analysis. All samples were processed in a national central lab in Beijing (medical laboratory accreditation certificate ISO 15189:2007) using automatic analyzer, with strict quality control.

Hemoglobin A1c (HbA1c) was measured in whole blood with an automated glycohemoglobin analyzer by using high-performance liquid chromatography system (model HLC-723 G7; Tosoh Corporation, Tokyo, Japan). HbA1c is used as a marker of prediabetes or diabetes (values $25.7 \%$ ) according to an International Expert Committee.(12) We also conducted comparative sensitivity analyses using fasting glucose (Supplemental Tables 2 and 3, Supplemental Figure 1). Plasma triglyceride and HDL-C were measured by using the glycerol-phosphate oxidase method, the PEG-modified enzyme HDL-C assay by determiner regents (Kyowa Medex Co., Ltd, Tokyo, Japan) on the Hitachi 7600 automated analyzer (Hitachi Inc., Tokyo, Japan). The International Diabetes Federation (IDF) criteria 
for adults(13) is used for indicators of dyslipidemia risk (high triglycerides: $\geq 150 \mathrm{mg} / \mathrm{dL}$; low HDL-C: (male: $<40 \mathrm{mg} / \mathrm{dL}$; female $<50 \mathrm{mg} / \mathrm{dL}$ ) or taking lipid lowering medication. High-sensitivity C-reactive protein (hsCRP), using the immunoturbidimetric method with Denka Seiken, Japan reagents (Hitachi 7600 automated analyzer, Hitachi Inc., Tokyo, Japan) is used as a marker of inflammation, with values $\geq 3 \mathrm{mg} / \mathrm{dL}$ indicating high risk.(14) Elevated hsCRP is a strong independent risk factor for future cardiovascular events, regardless of the underlying condition.(15)

Systolic (SBP) and diastolic (DBP) blood pressure were measured on the right arm, using mercury sphygmomanometers with appropriate cuff sizes. Measures were collected in triplicate after a 10 minute seated rest and the mean of the three measurements used in analyses. Risk was determined as systolic blood pressure (SBP) $\geq 130 \mathrm{mmHg} /$ diastolic blood pressure (DBP) $\geq 85 \mathrm{mmHg}$ according to the IDF cut point for pre-hypertension or taking blood pressure medication.(16)

Overweight and Abdominal Obesity-Height was measured without shoes to the nearest $0.2 \mathrm{~cm}$ using a portable SECA stadiometer, and weight was measured without shoes and in light clothing to the nearest $0.1 \mathrm{~kg}$ on a calibrated beam scale. Overweight was defined using the recommended Asian BMI cut point of $23 \mathrm{~kg} / \mathrm{m}^{2}$, with non-overweight $<23 \mathrm{~kg} / \mathrm{m}^{2}$.(17) We also conducted comparative sensitivity analyses using the WHO BMI $\geq 25 \mathrm{~kg} / \mathrm{m}^{2}$ cut point(18) (Supplemental Tables 4 and 5, Figure 2). Abdominal obesity was classified using waist circumference, which was measured with a SECA tape measure midway between the between the lower rib margin and the iliac crest. The IDF recommends sex and ethnic-specific waist circumference cut points, thus the Asian cut point for abdominal obesity for adults (waist circumference: men $\$ 90 \mathrm{~cm}$; women $\geq 80 \mathrm{~cm}$ ) was used. (13) Abdominal obesity is associated with cardiometabolic risk, independent of BMI,(19) particularly in individuals with normal BMI. $(20,21)$

Metabolically Healthy and Overweight Categories-Cardiometabolic risk is defined as having elevated values of one or more of the following five risk factors: inflammation (hsCRP $\geq 3 \mathrm{mg} / \mathrm{L}$ ), pre-hypertension or hypertension (SBP/DBP $\geq 130 / 85 \mathrm{mmHg}$ ), high triglycerides (triglycerides: $\geq 150 \mathrm{mg} / \mathrm{dL}$ ), low HDL-C: (male: $<40 \mathrm{mg} / \mathrm{dL}$; female $<50 \mathrm{mg}$ / $\mathrm{dL}$ ), and pre-diabetes or diabetes (HbA1c $\geq 5.7 \%)$. Patterns of risk associated with overweight were used to define the following four categories: "metabolically healthy, nonoverweight" (BMI $<23 \mathrm{~kg} / \mathrm{m}^{2}$, no risk factors); "metabolically healthy, overweight" (BMI $\geq$ $23 \mathrm{~kg} / \mathrm{m}^{2}$, no risk factors); "metabolically at risk, non-overweight" (BMI $<23 \mathrm{~kg} / \mathrm{m}^{2}, \geq 1$ risk factor); and "metabolically at risk, overweight" (BMI $\geq 23 \mathrm{~kg} / \mathrm{m}^{2}, \geq 1$ risk factor). Inflammation was considered part of the cardiometabolic risk profile since in developing countries, high rates of obesity occur alongside high rates of infectious disease and malnutrition, resulting in dual burden of obesity-related and pathogen-related inflammation(22) and future cardiovascular disease.(23) Abdominal obesity was not considered in the definitions of these phenotypes due to its covariation with BMI and conceptual interest in the association of abdominal obesity with these phenotypes.

Control variables-Age was recorded as the respondent's age on the date of exam, ranging from 18 to 98 years and categorized into the following categories: $18-30,30-40$, 
$40-50,50-60,60-70$, and $>70$ years. Number of cigarettes per day were reported and categorized as currently smoking one or more cigarettes per day. Alcohol was reported as number of alcoholic beverages consumed per week across a variety of beverage types, categorized according to consumption of $\geq 1$ servings of alcohol per day. Physical activity was reported using a detailed 7-day physical activity recall instrument and leisure activities assigned Metabolic Equivalent (MET) values using the Compendium of Physical activity, (24) which has been used in international contexts, including China.(25) MET values were multiplied by the time spent in each activity and categorized according to $\geq 27$ METs per week, equivalent to $\geq 1$ hour per week of moderate-intensity, such as brisk walking. Urbanicity was defined using a multidimensional 12 component urbanization index capturing community-level physical, social, cultural, and economic environments and represents the heterogeneity otherwise missed in an urban/rural measure based only on population density.(26) Tertiles of the 100 value index represent low, medium, and high urbanicity. Household assets and household income were used to derive income; tertiles represent low, medium, and high income.(27)

\section{Statistical Analyses}

We conducted statistical analyses using Stata (Release 11.0, Stata Corporation, College Station, TX). In descriptive analyses, markers are compared across overweight status (percentages for categorical variables, means for continuous variables). A series of models were used to examine overweight (BMI $\geq 23 \mathrm{~kg} / \mathrm{m} 2$ ) and cardiometabolic risk patterning ( $\geq 1$ of the following risk factors: inflammation, pre-hypertension or hypertension, high triglycerides, low HDL-C, pre-diabetes or diabetes) and assesses the independent and interactive effects of overweight and abdominal obesity (waist circumference: men $>90 \mathrm{~cm}$; women $>80 \mathrm{~cm}$ ). First, multinomial regression models predicting risk of falling into one of four categories: 1) "metabolically healthy, normal weight", 2) "metabolically healthy, overweight", 3) "metabolically at risk, overweight," and 4) "metabolically at risk, normal weight"[referent], with abdominal obesity as the main exposure were used to test the hypothesis that abdominal obesity would differentially predict membership into one of the four risk categories. Second, a series of five separate logistic regression models for each outcome (inflammation, pre-hypertension or hypertension, high triglycerides, low HDL-C, pre-diabetes or diabetes) were used sequentially to test the hypotheses that: 1) overweight (first main exposure) differentially associates with each cardiometabolic risk factor, and 2) abdominal obesity (second main exposure), controlling for overweight, confers differential risk for each cardiometabolic marker. Third, five separate and sequential logistic regression models for each outcome (inflammation, pre-hypertension or hypertension, high triglycerides, low HDL-C, pre-diabetes or diabetes) with four main exposure variables: 1) overweight with abdominal obesity; 2) overweight with no abdominal obesity; 3) nonoverweight with abdominal obesity; and 4) non-overweight with no abdominal obesity [referent], were used to test the hypothesis that there is an interactive effect between overweight and abdominal obesity, with overweight and abdominal obese individuals at highest risk. All models were sex-stratified and included the following covariates: age, smoking, alcohol consumption, physical activity, urbanicity, and income. While model building suggested minimal change in effect of the main exposure with the addition of smoking, alcohol consumption, physical activity, urbanicity, and income, these covariates 
were retained on conceptual grounds. Two forms of sensitivity analyses were performed: (1) using the BMI $\geq 25 \mathrm{~kg} / \mathrm{m}^{2}$ cut point rather than the more stringent Asian BMI $\geq 23 \mathrm{~kg} / \mathrm{m}^{2}$ cut point, and (2) using impaired fasting glucose ( $\geq 100 \mathrm{mg} / \mathrm{dL}$ ) as opposed to HbA1c (prediabetes or diabetes) as the indicator of diabetes risk. Results from both sensitivity analyses are shown in the Supplemental Materials.

\section{RESULTS}

While approximately half of the sample was overweight, $78 \%$ had elevated values of one or more cardiometabolic risk factors ( $49 \%$ had more than two) (Table 1). The proportion with pre-hypertension or hypertension $(42.8 \%)$ and pre-diabetes or diabetes $(38.1 \%)$ was particularly high. Rates of isolated hypertension risk (9.9\%) and pre-diabetes or diabetes (7.7\%) were roughly twice as high as isolated high triglycerides, low HDL-C, and inflammation, which were around 4\%. In addition, mean waist circumference of the metabolically healthy non-overweight group were on average $16 \mathrm{~cm}$ less than that of the metabolically at risk overweight group, $10 \mathrm{~cm}$ less than the metabolically healthy overweight, and $3 \mathrm{~cm}$ less than the metabolically at risk non-overweight group.

All cardiometabolic risk factors were higher among the overweight (Table 2). The proportion of overweight and non-overweight individuals with inflammation, prehypertension or hypertension, and pre-diabetes or diabetes increased with age, whereas rates of both high triglycerides and low HDL-C were highest in the early adult years for men and HDL-C was relatively stable among women. Relative to the other risk factors, differences in hypertension risk by overweight status were not as large. Mean continuous values for each marker are shown in Supplemental Table 1.

A comparatively large proportion of the sample across all age groups was "metabolically at risk normal weight", notably $42 \%$ of the total sample aged 18-30 (Figure 1). There were very few "metabolically healthy overweight" individuals and the proportion of the sample considered metabolically healthy (regardless of overweight status) declined considerably with age. Findings were similar across low, medium, and high urbanicity.

We used multinomial regression models to test the hypothesis that abdominal obesity differentially predicted membership into one of the four categories of obesity with and without metabolic risk (Table 3). The odds of being in the "metabolically at risk overweight" compared to the metabolically healthy, non-overweight group was dramatically elevated with abdominal obesity (men, Odds Ratio (OR): 39.06, 95\% Confidence Interval (CI): 23.47-65.00; women, OR: 22.26, CI: 17.49-28.33). Abdominal obesity was also predictive of having high metabolic risk among both non-overweight and overweight individuals.

We used cardiometabolic risk factor-specific logistic regression models to examine the association between overweight and each risk factor, with and without control for abdominal obesity (Set A, models 1 and 2). One set of models included overweight exposure only (Table 4, Column 2), a second set of models included both overweight and abdominal obesity as exposure variables (Table 4, Column 3 ). As expected, overweight independently 
increased the risk of each cardiometabolic marker in men and women, with variation across indicators. Independent of overweight status, abdominal obesity conferred risk for all cardiometabolic indicators.

A third set of cardiometabolic risk factor-specific logistic regression models was used to examine the interactive association between overweight and abdominal obesity with each risk factor (Set B models): 1) overweight with abdominal obesity, 2) overweight with no abdominal obesity, and 3) non-overweight with abdominal obesity relative to 4) nonoverweight with no abdominal obesity. Overweight individuals with abdominal obesity were at comparatively high cardiometabolic risk, with variation across pre-hypertension or hypertension, pre-diabetes or diabetes, inflammation and dyslipidemia (Table 4, Column 6). In the majority of cases, there was close to or above a threefold higher risk with the combination of overweight and abdominal obesity relative to non-overweight and no abdominal obesity.

\section{Sensitivity Analysis Results}

Results were remarkably similar when impaired fasting glucose ( $\geq 100 \mathrm{mg} / \mathrm{dL}$ ) as opposed to HbA1c (pre-diabetes or diabetes) was used as the indicator of diabetes risk (Supplemental Tables 2 and 3, Supplemental Figure 1). Further, results were similar when overweight was defined using BMI $\geq 25 \mathrm{~kg} / \mathrm{m}^{2}$ cut point(18) (Supplemental Tables 4 and 5), but with a considerably larger portion of the population considered "metabolically at risk nonoverweight" (Supplemental Figure 2). Relative to the BMI $\geq 23 \mathrm{~kg} / \mathrm{m}^{2}$ cut point, using the BMI $25 \mathrm{~kg} / \mathrm{m}^{2}$ cut point suggests that abdominal obesity has the same (albeit stronger) positive association with the metabolically at risk overweight.

\section{DISCUSSION}

Over three-quarters of this national Chinese sample had elevated levels of at least one cardiometabolic risk factor. Even in young adults, only half were "metabolically healthy" at 18-30 years of age, but this decreased to $6 \%$ in the 60-70 year olds. Findings were similar across low, medium, and high urbanicity, indicating high prevalence of metabolic abnormalities even in the rural Chinese population, according to the IDF criteria for metabolic risk. Just over 5\% of the overweight adults had no risk factors. Among the nonoverweight adults, $33.2 \%$ had at least one risk factor. Rates of isolated high triglycerides, low HDL-C, and inflammation were relatively low. The predominant risk factor was hypertension risk, which increased dramatically with age, underlying the decline with age in the proportion of adults considered metabolically healthy. While we cannot rule out the fact that the high rates of hypertension risk might be a result of the normal increase in blood pressure with age, the high prevalence of metabolic abnormalities among the 18-30 year old group, particularly in low HDL-C and high triglycerides, is notable. Our findings highlight the importance of screening in the general population, given the large portion of nonoverweight individuals with cardiometabolic risk. This should be at the forefront in China's efforts to prevent and control non-communicable diseases.

Previous research suggests that hypertension risk is particularly high in China and is found at low BMI levels in Asians relative to other subpopulations. $(5,28,29)$ A recent paper on 
diabetes in China documented high diabetes prevalence,(30) although that sample does not represent poor, less urbanized areas, as well as the CHNS. While HDL-C tends to be relatively low in Asians,(31) rates of low HDL-C were not extraordinary in these Chinese adults. Inflammation, a risk factor for type 2 diabetes and cardiovascular disease,(32-34) is associated with insulin resistance and cardiometabolic risk in the absence of obesity.(35) The observed findings suggest that inflammation increases with age across all adults, which is common in developing countries where high rates of obesity occur alongside high rates of infectious disease and malnutrition, resulting in dual burden of obesity-related and pathogen-related inflammation.(22) Elevated hsCRP is a strong independent risk factor for future cardiovascular events, regardless of the underlying condition.(15) For example, risk may be present even if the underlying etiology relates to periodontal disease, which is an independent risk factor for coronary heart disease.(36) Despite the sex difference in abdominal obesity (largely due to the lower threshold for abdominal obesity in women (80 $\mathrm{cm})$ versus men $(90 \mathrm{~cm})$, inflammation rates were similar. The combined association between overweight and abdominal obesity with cardiometabolic risk factors suggests a multiplicative effect on cardiometabolic risk, with risk that is magnified in overweight individuals, even after controlling for smoking, alcohol consumption, total physical activity, urbanicity, and income.

Most cardiometabolic screening is aimed at individuals above a given threshold of body mass, despite the fact that elevated cardiometabolic risk is common in the general population.(37) The observed high risk among non-overweight Chinese, and the increase in risk with age, implies that screening for cardiometabolic risk should occur across all adults, regardless of weight status. Further, the high prevalence of pre-hypertension and hypertension, pre-diabetes or diabetes, high triglycerides, low HDL-C, and inflammation risk even below the BMI $23 \mathrm{~kg} / \mathrm{m}^{2}$ threshold supports a lower BMI cut point for the Chinese population. $(4,17)$

China has had a history of undernutrition followed by the most rapid increase in obesity and related diseases worldwide, with differential rates across rural and urban areas. $(2,38)$ In the last decade, changes in diet and activity in China have occurred more rapidly than previously recorded for any country,(2) including increased intake of edible oil, fried foods, animal source foods and snacking, as well as declining occupational, domestic, and travel activity expenditure and increasing TV time. $(25,39)$ The observed findings fit with the trend towards less healthier lifestyles, and indicate elevated cardiometabolic risk even in the rural areas of China.

There are a few limitations to this analysis. This cross-sectional analysis does not examine trends in risk or determinants of cardiometabolic risk. The main objective of this study was to determine the prevalence of "metabolically healthy overweight" and "metabolically at risk non-overweight" in Chinese adults and to examine patterns of risk by age and gender. As such, the analytic strategy was designed to adjust for key covariates, rather than undertaking causal modeling of these relationships. The use of conventional, anthropometric and cardiometabolic cut points do not capture differences in cardiometabolic risk that exist on the continuum of BMI and waist circumference. Likewise, cut points do not capture the complex process of cardiometabolic risk However, cut points are needed for clinical 
guidance and there is rationale for use of Asian-specific BMI cut points based on higher risk at lower BMI.(4, 17, 40) Finally, BMI does not discriminate between lean and adipose tissue and waist circumference is an indicator of both the subcutaneous and visceral adipose tissue compartments,(41) thus we cannot distinguish the two.

In summary, findings suggest a high prevalence of cardiometabolic risk in China that increases with age. The majority of the sample is considered metabolically at risk, with similar patterns of risk across urbanicity. Rates of risk increase dramatically with age and with overweight and abdominal obesity. The combined association between overweight and abdominal obesity with cardiometabolic risk factors, even after controlling for smoking, alcohol consumption, total physical activity, urbanicity, and income, suggest extraordinary burden of future cardiovascular disease with population increases in weight and abdominal obesity - a major clinical concern. Screening for cardiometabolic-related outcomes dependent upon overweight will likely miss a large portion of the Chinese at-risk population and has implications for other Asian populations.

\title{
Supplementary Material
}

Refer to Web version on PubMed Central for supplementary material.

\section{ACKNOWLEDGEMENT}

\begin{abstract}
We thank Ms. Frances Dancy, BS, UNC Carolina Population Center for her helpful administrative assistance, Jim Terry AB, Phil Bardsley PhD, and Dan Blanchette BA for programming and technical support. P.G.L. and L.S.A. contributed to study design, P.G.L., and L.S.A. contributed to data analysis, and all authors contributed to writing of the manuscript. P.G.L. had full access to all the data in the study and takes responsibility for the integrity of the data and the accuracy of the data analysis.

Funding Sources

This work was supported by NIH: NIDDK (R21DK089306) and NHLBI (R01-HL108427). NIH had no role in the design and conduct of the study; collection, management, analysis, and interpretation of the data; and preparation, review, or approval of the manuscript. This research uses data from the China Health and Nutrition Survey, funded by NIH: NICHD (R01-HD30880), although no direct support was received from grant for this analysis. Dr. Meigs is supported by National Institute for Diabetes and Digestive and Kidney Diseases (NIDDK) K24DK080140.
\end{abstract}

\section{REFERENCES}

1. Wildman RP, Muntner P, Reynolds K, et al. The Obese Without Cardiometabolic Risk Factor Clustering and the Normal Weight With Cardiometabolic Risk Factor Clustering: Prevalence and Correlates of 2 Phenotypes Among the US Population (NHANES 1999-2004). Arch Intern Med. 2008; 168(15):1617-1624. [PubMed: 18695075]

2. Popkin BM. Will China's nutrition transition overwhelm its health care system and slow economic growth? Health Aff. 2008; 27(4):1064-1076.

3. He J, Gu D, Wu X, et al. Major Causes of Death among Men and Women in China. N Engl J Med. 2005; 353(11):1124-1134. [PubMed: 16162883]

4. Deurenberg P, Deurenberg-Yap M, Guricci S. Asians are different from Caucasians and from each other in their body mass index/body fat per cent relationship. Obes Rev. 2002; 3(3):141-146. [PubMed: 12164465]

5. Bell A, Adair LS, Popkin BM. Ethnic differences in the association between body mass index and hypertension. Am J Epidemiol. 2002; 155(4):346-353. [PubMed: 11836199]

6. Chan JCN, Malik V, Jia W, et al. Diabetes in Asia: Epidemiology, Risk Factors, and Pathophysiology. JAMA. 2009; 301(20):2129-2140. [PubMed: 19470990] 
7. Gu D, Reynolds K, Wu X, et al. Prevalence of the metabolic syndrome and overweight among adults in China. Lancet. 2005; 365(9468):1398-1405. [PubMed: 15836888]

8. Wildman RP, McGinn AP, Lin J, Wang D, Muntner P, Cohen HW, et al. Cardiovascular disease risk of abdominal obesity vs. metabolic abnormalities. Obesity. 2011; 19(4):853-860. [PubMed: 20725064]

9. Lloyd-Jones DM, Wilson PW, Larson MG, et al. Framingham risk score and prediction of lifetime risk for coronary heart disease. Am J Cardiol. 2004; 94(1):20-24. [PubMed: 15219502]

10. Tzoulaki I, Liberopoulos G, Ioannidis JP. Assessment of claims of improved prediction beyond the Framingham risk score. JAMA. 2009; 302(21):2345-2352. [PubMed: 19952321]

11. Popkin BM, Du S, Zhai F, Zhang B. Cohort Profile: The China Health and Nutrition Survey- monitoring and understanding socio-economic and health change in China, 1989-2011. Int J Epidemiol. 2010; 39(6):1435-1440. [PubMed: 19887509]

12. International Expert Committee. International Expert Committee Report on the Role of the A1C Assay in the Diagnosis of Diabetes. Diabetes Care. 2009; 32(7):1327-1334. [PubMed: 19502545]

13. Alberti KGMM, Zimmet P, Shaw J. The metabolic syndrome--a new worldwide definition. Lancet. 2005; 366(9491):1059-1062. [PubMed: 16182882]

14. Pearson TA, Mensah GA, Alexander RW, Anderson JL, Cannon RO, Criqui M, et al. Markers of Inflammation and Cardiovascular Disease. Circulation. 2003; 107(3):499-511. [PubMed: 12551878]

15. Buckley DI, Fu R, Freeman M, Rogers K, Helfand M. C-Reactive Protein as a Risk Factor for Coronary Heart Disease: A Systematic Review and Meta-analyses for the U.S. Preventive Services Task Force. Annals of Internal Medicine. 2009; 151(7):483-495. [PubMed: 19805771]

16. National High Blood Pressure Education Program Working Group on High Blood Pressure in Children and Adolescents. The Fourth Report on the Diagnosis, Evaluation, and Treatment of High Blood Pressure in Children and Adolescents. Pediatrics. 2004; 114(2):555-576. [PubMed: 15286277]

17. WHO Expert Consultation. WHO Expert Consultation. Appropriate body mass index for Asian populations and its implications for policy and intervention strategies. Lancet. 2004; 363(9403): 157-163. [PubMed: 14726171]

18. WHO Expert Committee. Physical Status: The Use and Interpretation of Anthopometry: Report of a WHO Expert Committee. Geneva: World Health Organization; p. 1-854.

19. Demerath EW, Reed D, Rogers N, et al. Visceral adiposity and its anatomical distribution as predictors of the metabolic syndrome and cardiometabolic risk factor levels. Am J Clin Nutr. 2008; 88(5):1263-1271. [PubMed: 18996861]

20. Balkau B, Deanfield JE, Despres J-P, et al. International Day for the Evaluation of Abdominal Obesity (IDEA): A Study of Waist Circumference, Cardiovascular Disease, and Diabetes Mellitus in 168000 Primary Care Patients in 63 Countries. Circulation. 2007; 116(17):1942-1951. [PubMed: 17965405]

21. Lee CMY, Huxley RR, Wildman RP, Woodward M. Indices of abdominal obesity are better discriminators of cardiovascular risk factors than BMI: a meta-analysis. J Clin Epid. 2008; 61(7): 646-653.

22. McDade TW, Rutherford JN, Adair L, Kuzawa C. Adiposity and pathogen exposure predict Creactive protein in Filipino women. J Nutr. 2008; 138(12):2442-2447. [PubMed: 19022970]

23. Ridker PM, Cook N. Clinical usefulness of very high and very low levels of C-reactive protein across the full range of Framingham Risk Scores. Circulation. 2004; 109(16):1955-1959. [PubMed: 15051634]

24. Ainsworth BE, Haskell WL, Whitt MC, et al. Compendium of physical activities: an update of activity codes and MET intensities. Med Sci Sports Exerc. 2000; 32(9 Suppl):S498-S504. [PubMed: 10993420]

25. Monda KL, Adair LS, Zhai F, Popkin BM. Longitudinal relationships between occupational and domestic physical activity patterns and body weight in China. Eur J Clin Nutr. 2008; 62(11):13181325. [PubMed: 17637599] 
26. Jones-Smith JC, Popkin BM. Understanding community context and adult health changes in China: development of an urbanicity scale. Soc Sci \& Med. 2010; 71(8):1436-1446. [PubMed: 20810197]

27. Doak C, Adair L, Bentley M, Fengying Z, Popkin B. The underweight/overweight household: an exploration of household sociodemographic and dietary factors in China. Publ Health Nutr. 2002; 5(1A):215-221.

28. Eastern Stroke and Coronary Heart Disease Collaborative Research Group. Blood pressure, cholesterol, and stroke in eastern Asia. Lancet. 1998; 352(9143):1801-1807. [PubMed: 9851379]

29. Zhao D, Liu J, Wang W, et al. Epidemiological transition of stroke in China: twenty-one-year observational study from the Sino-MONICA-Beijing Project. Stroke. 2008; 39(6):1668-1674. [PubMed: 18309149]

30. Yang W, Lu J, Weng J, et al. Prevalence of diabetes among men and women in China. N Engl J Med. 2010; 362(12):1090-1101. [PubMed: 20335585]

31. Patel A, Huang KC, Janus ED, et al. Is a single definition of the metabolic syndrome appropriate?-A comparative study of the USA and Asia. Atherosclerosis. 2006; 184(1):225-232. [PubMed: 15935356]

32. Pradhan AD, Manson JE, Rifai N, Buring JE, Ridker PM. C-reactive protein, interleukin 6, and risk of developing type 2 diabetes mellitus. JAMA. 2001; 286(3):327-334. [PubMed: 11466099]

33. Pickup JC. Inflammation and activated innate immunity in the pathogenesis of type 2 diabetes. Diabetes Care. 2004; 27(3):813-823. [PubMed: 14988310]

34. Ford ES, Liu S, Mannino DM, Giles WH, Smith SJ. C-reactive protein concentration and concentrations of blood vitamins, carotenoids, and selenium among United States adults. Eur J Clin Nutr. 2003; 57(9):1157-1163. [PubMed: 12947436]

35. Ye X, Yu Z, Li H, Franco OH, Liu Y, Lin X. Distributions of C-reactive protein and its association with metabolic syndrome in middle-aged and older Chinese people. J Am Coll Cardiol. 2007; 49(17):1798-1805. [PubMed: 17466231]

36. Humphrey LL, Fu R, Buckley DI, Freeman M, Helfand M. Periodontal disease and coronary heart disease incidence: a systematic review and meta-analysis. J Gen Intern Med. 2008; 23(12):2079_ 2086. [PubMed: 18807098]

37. Ford ES, Giles WH, Dietz WH. Prevalence of the metabolic syndrome among US adults: findings from the third National Health and Nutrition Examination Survey. JAMA. 2002; 287(3):356-359. [PubMed: 11790215]

38. Van de Poel E, O'Donnell O, Van Doorslaer E. Are urban children really healthier? Evidence from 47 developing countries. Soc Sci Med. 2007; 65(10):1986-2003. [PubMed: 17698272]

39. Ng SW, Zhai F, Popkin BM. Impacts of China's edible oil pricing policy on nutrition. Soc Sci Med. 2008; 66(2):414-426. [PubMed: 17996345]

40. Odegaard AO, Pereira MA, Koh WP, et al. BMI all-cause and cause-specific mortality in Chinese Singaporean men and women: the Singapore Chinese health study. PLoS One. 2010;

5(11):e14000. [PubMed: 21085577] 


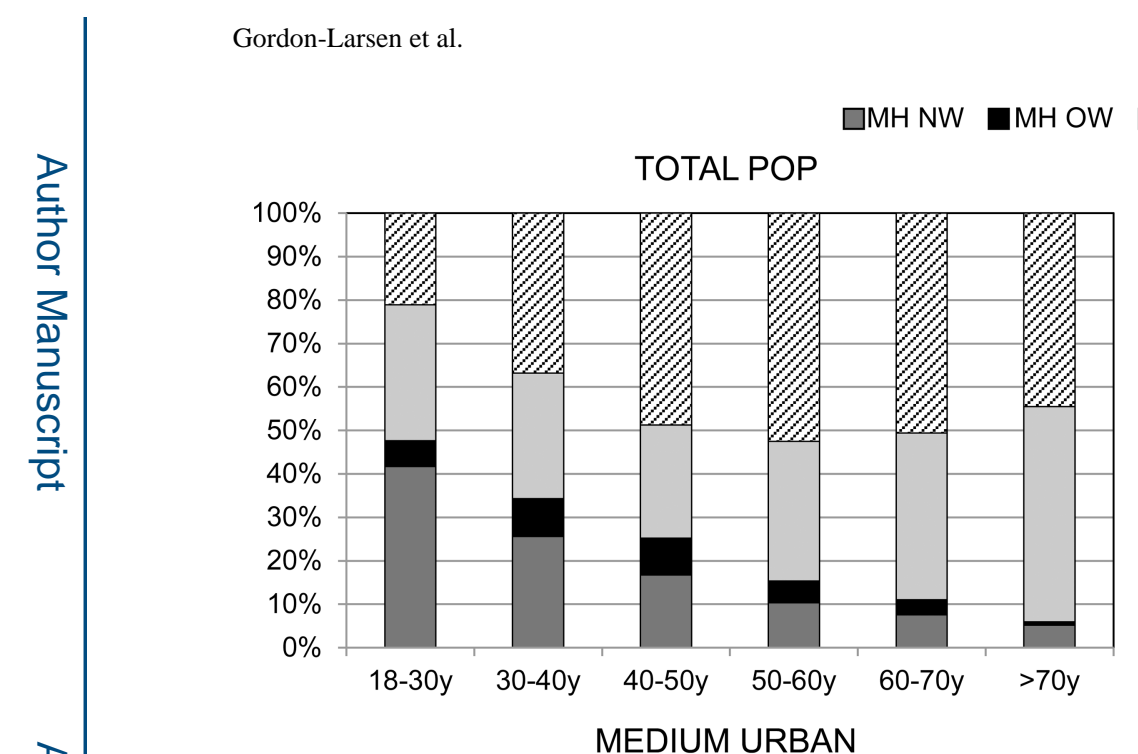

\section{DMAR NW छMAR OW}
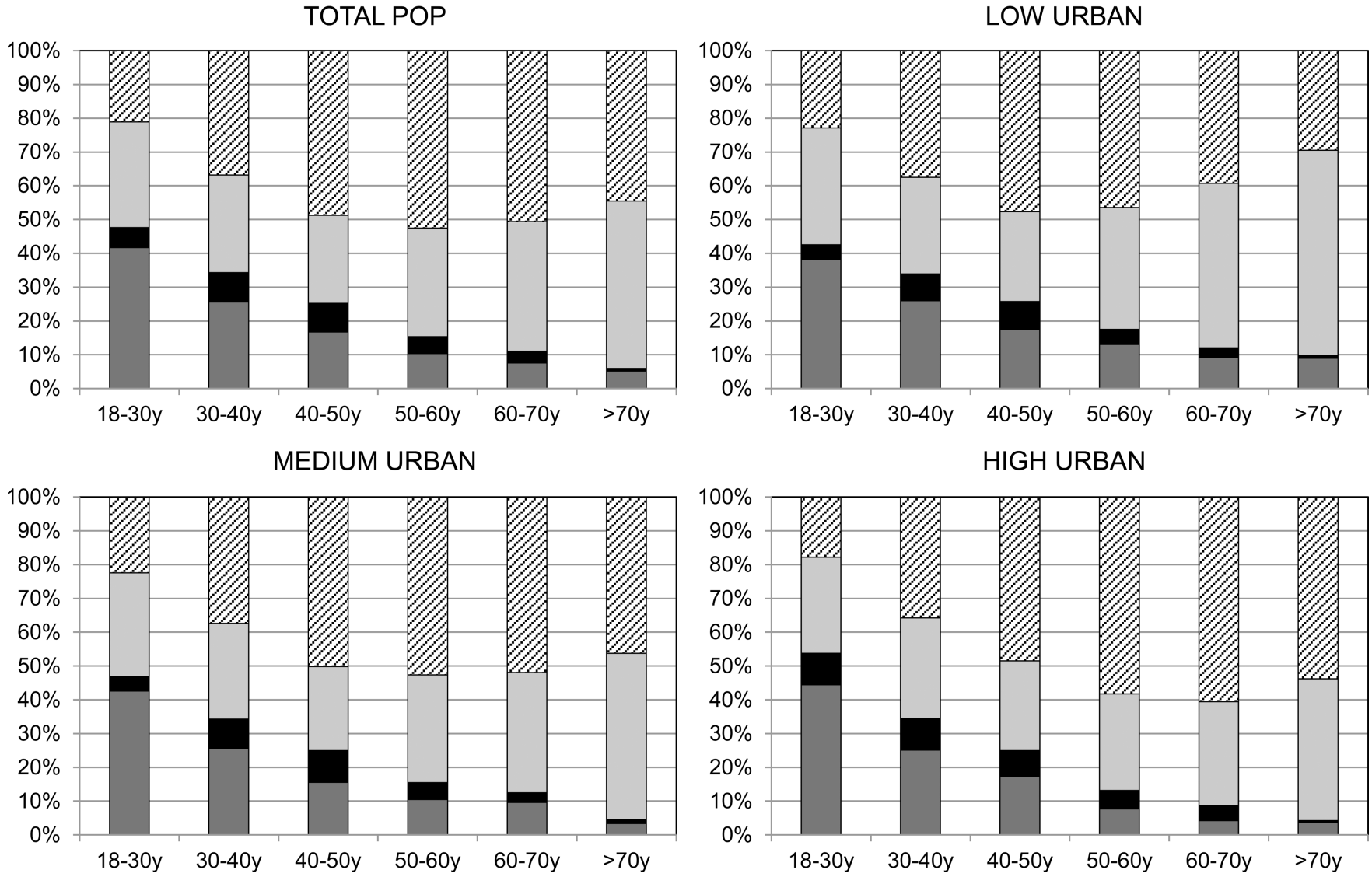

Figure 1. Concurrent Overweighta and Cardiometabolic Riskb, Total Population and Across Urbanicity

${ }^{\mathrm{a}}$ Overweight defined using the BMI $\geq 23 \mathrm{~kg} / \mathrm{m}^{2}$ cutpoint(17)

${ }^{b}$ Cardiometabolic Risk, defined as one or more of the following risk factors: dysglycemia (HbA1c $\geq 5.7 \%$ ) inflammation (hsCRP $\geq 3 \mathrm{mg} / \mathrm{L})$, pre-hypertension/hypertension (SBP/ $\mathrm{DBP} \geq 130 / 85 \mathrm{mmHg}$ ), high triglycerides ( $\geq 150 \mathrm{mg} / \mathrm{dL}$ ), low high-density lipoprotein cholesterol ( $<40 \mathrm{mg} / \mathrm{dL}$, men; $<50 \mathrm{mg} / \mathrm{dL}$, women).

NOTE: MH NW = Metabolically Healthy, Normal Weight

MH OW = Metabolically Healthy, Overweight

MAR NW = Metabolically at Risk, Normal Weight

MAR OW =Metabolically at Risk, Overweight 


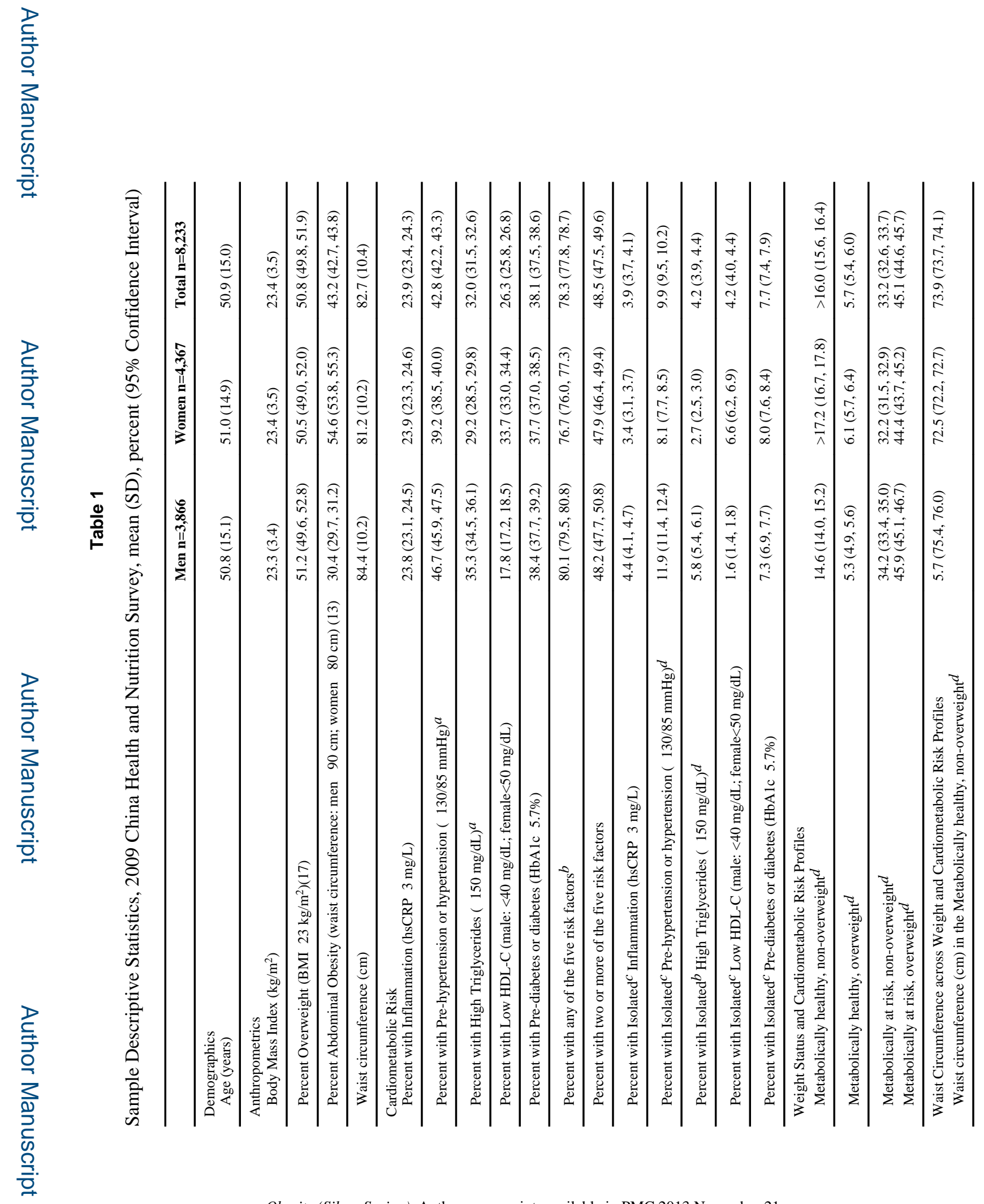

Obesity (Silver Spring). Author manuscript; available in PMC 2013 November 21. 


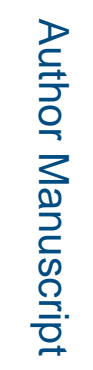

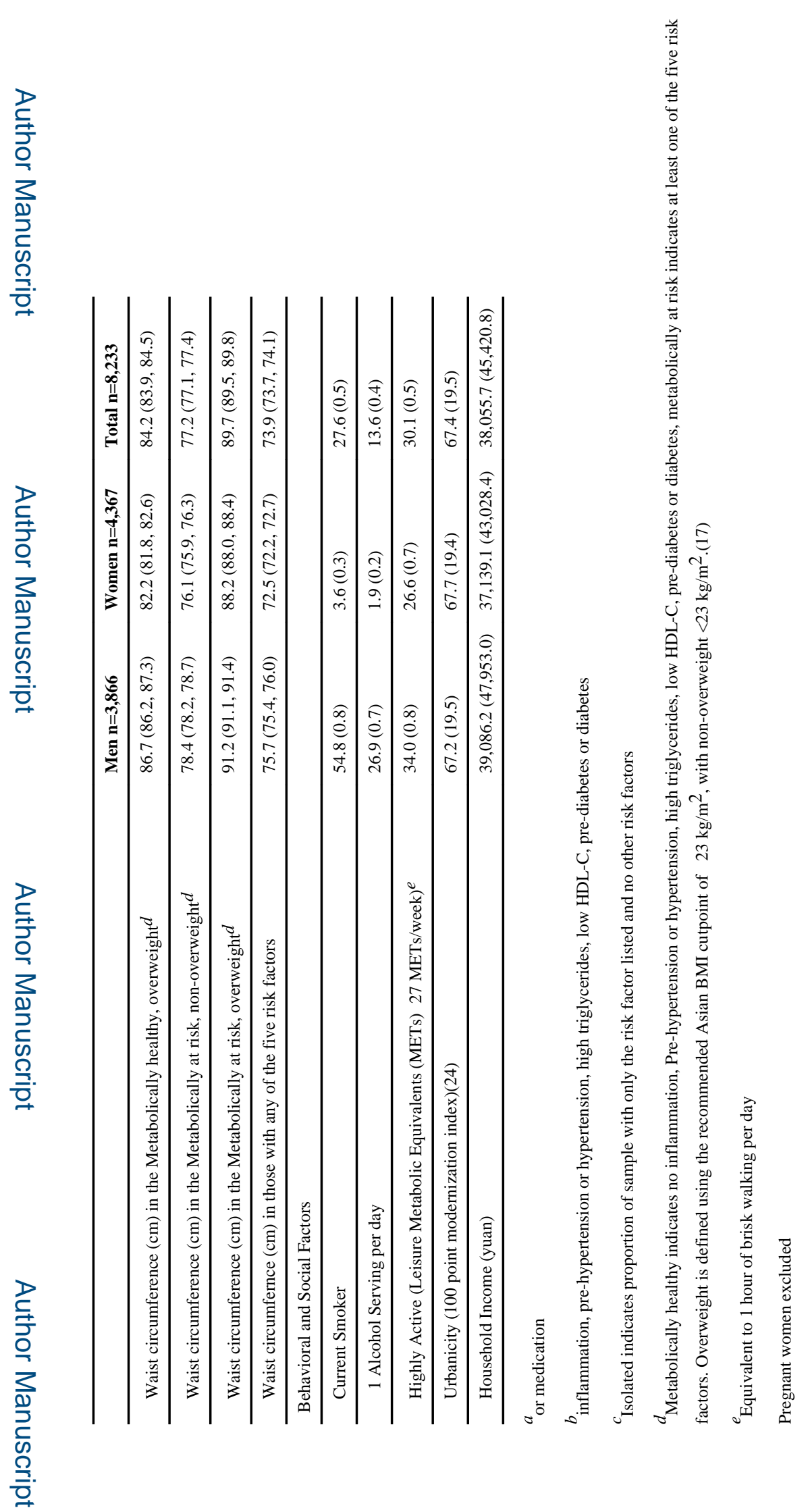

Obesity (Silver Spring). Author manuscript; available in PMC 2013 November 21. 


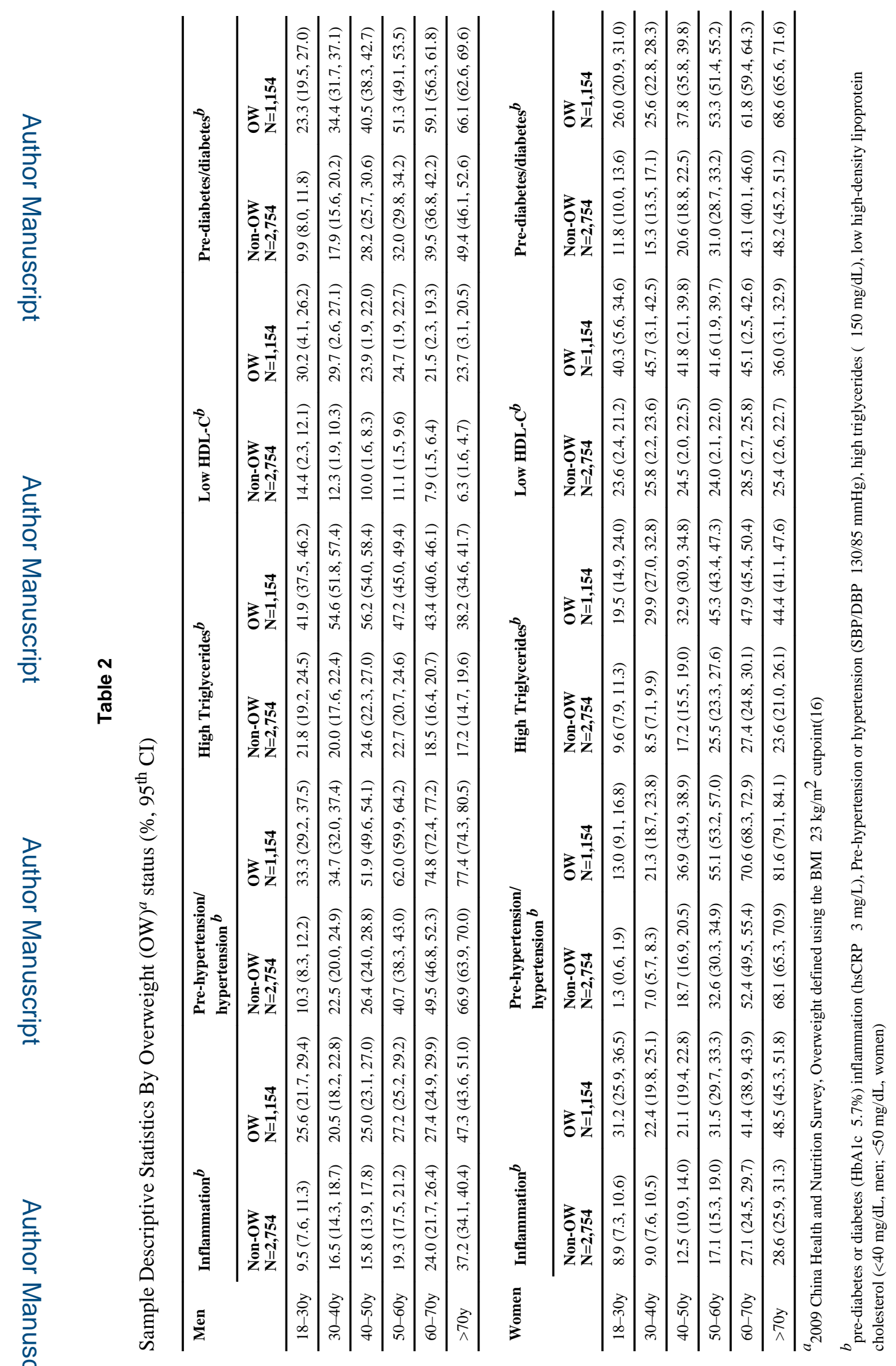

Obesity (Silver Spring). Author manuscript; available in PMC 2013 November 21. 


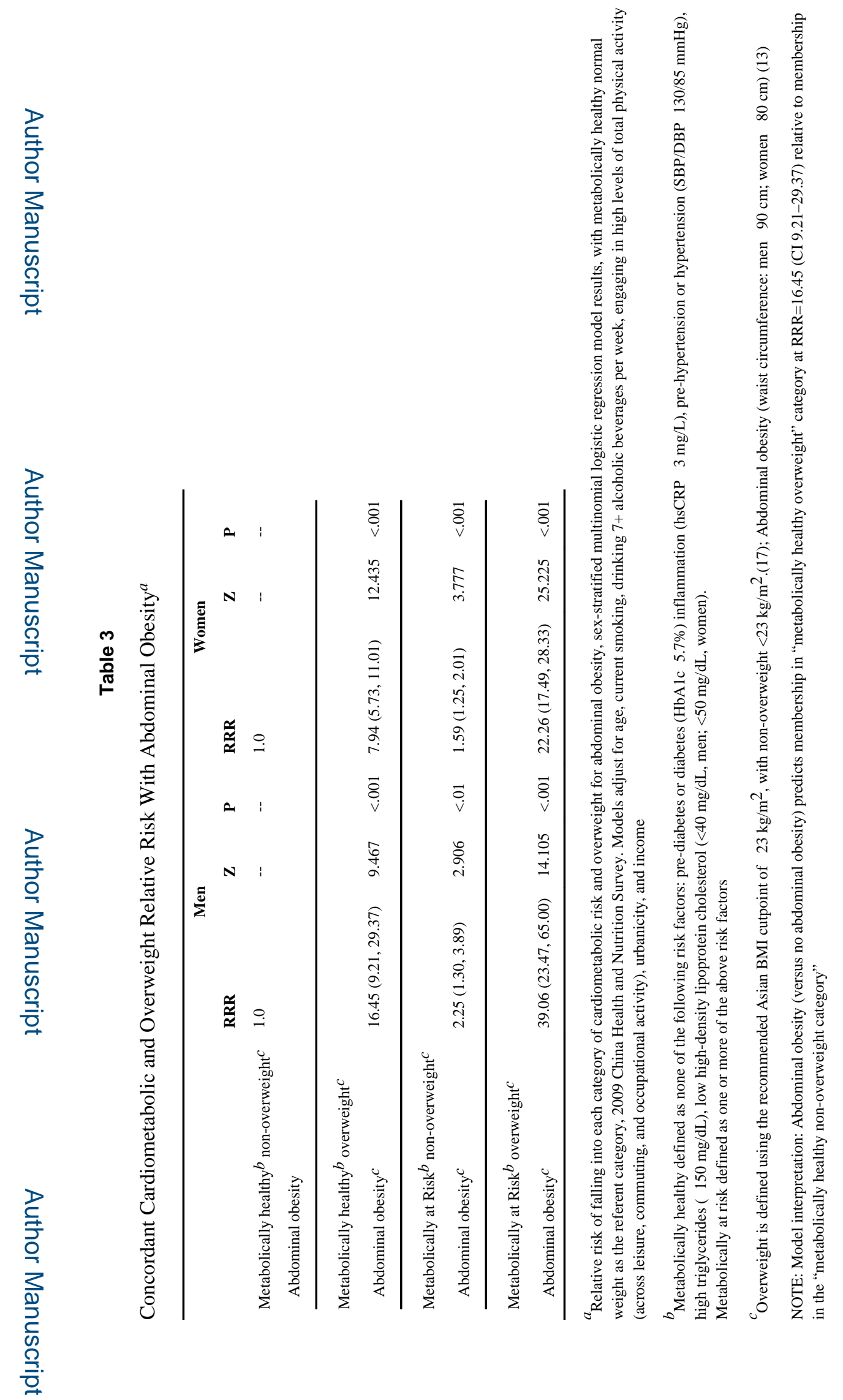

Obesity (Silver Spring). Author manuscript; available in PMC 2013 November 21. 


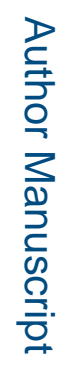

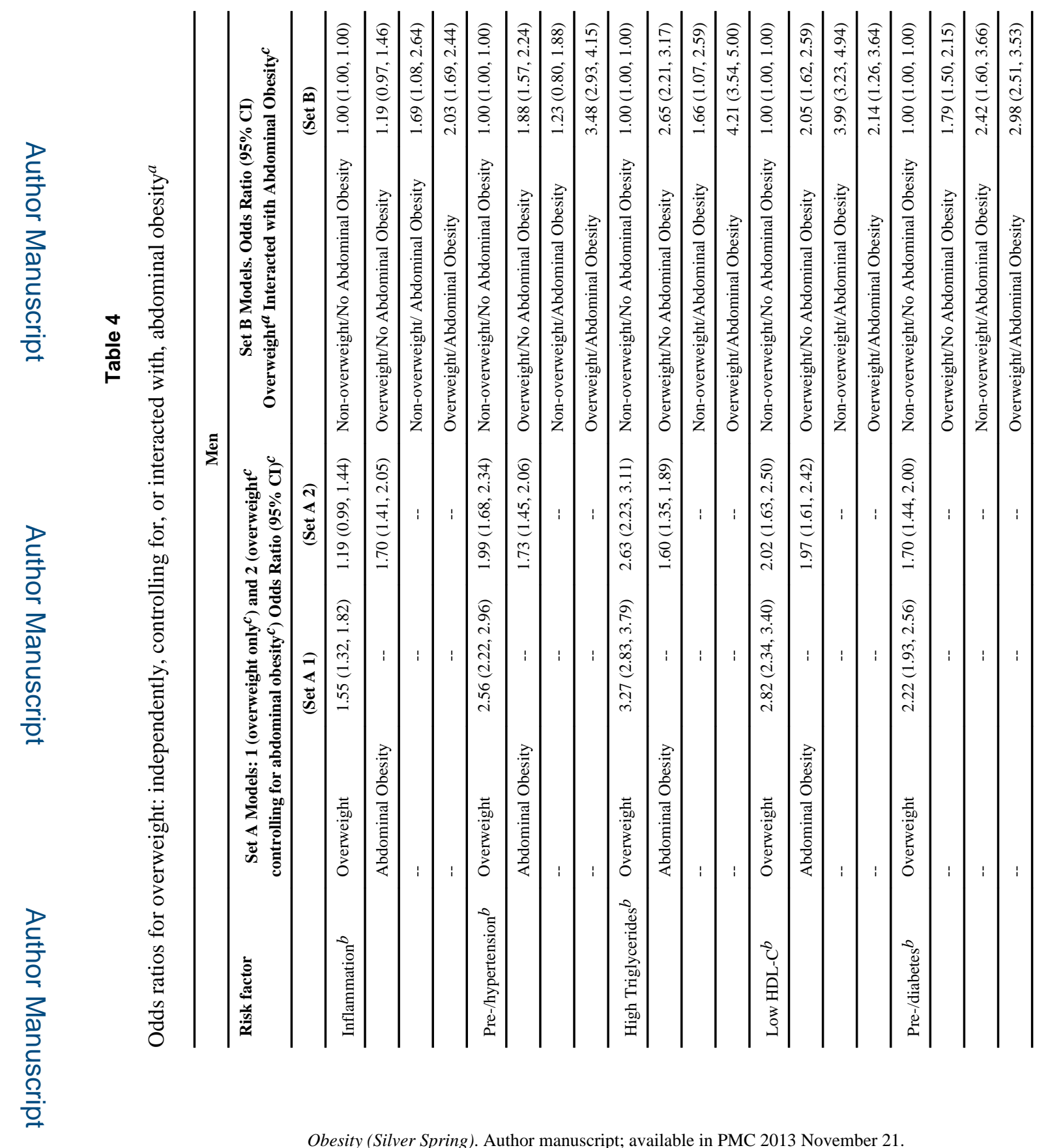




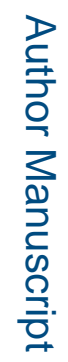

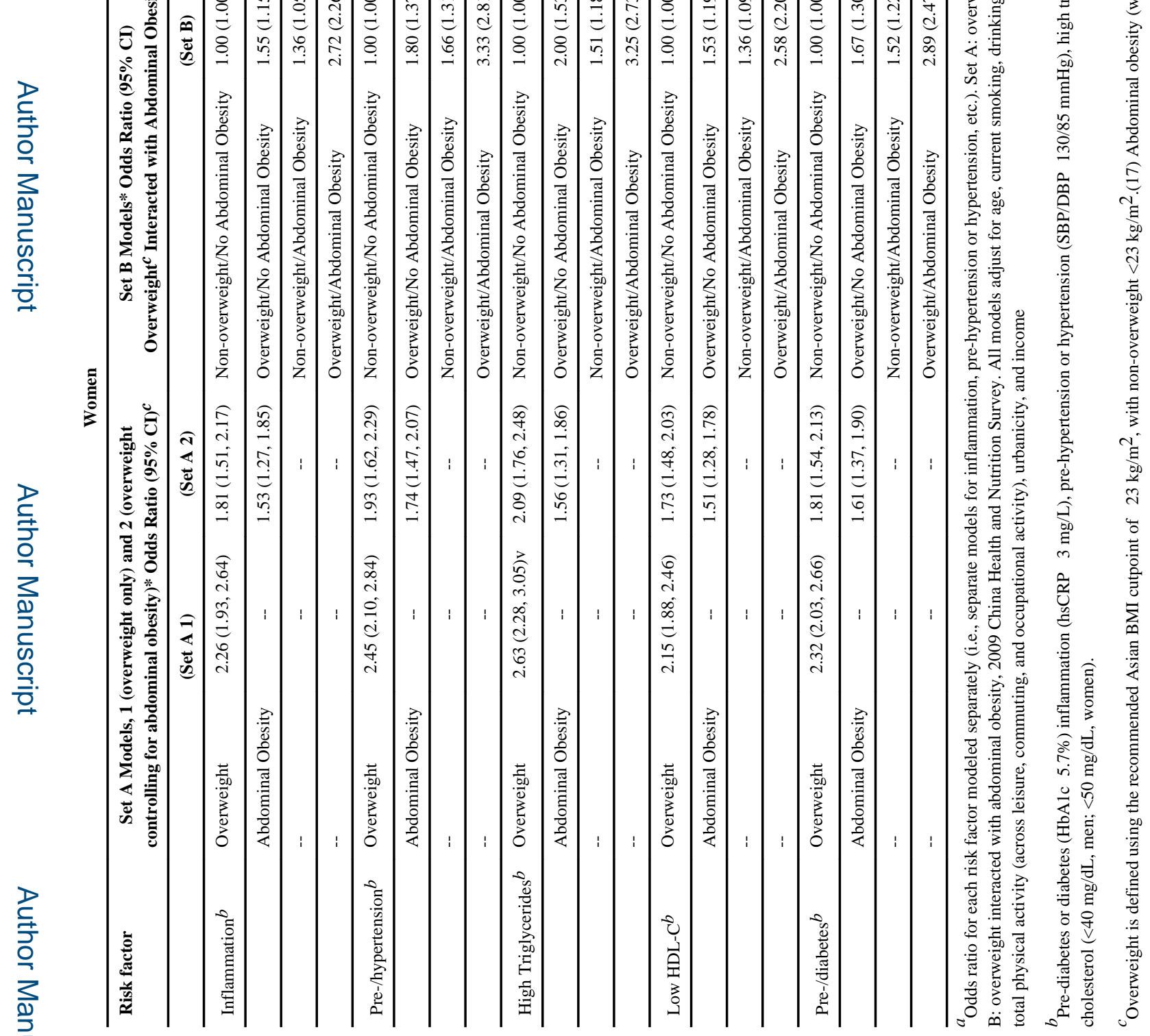

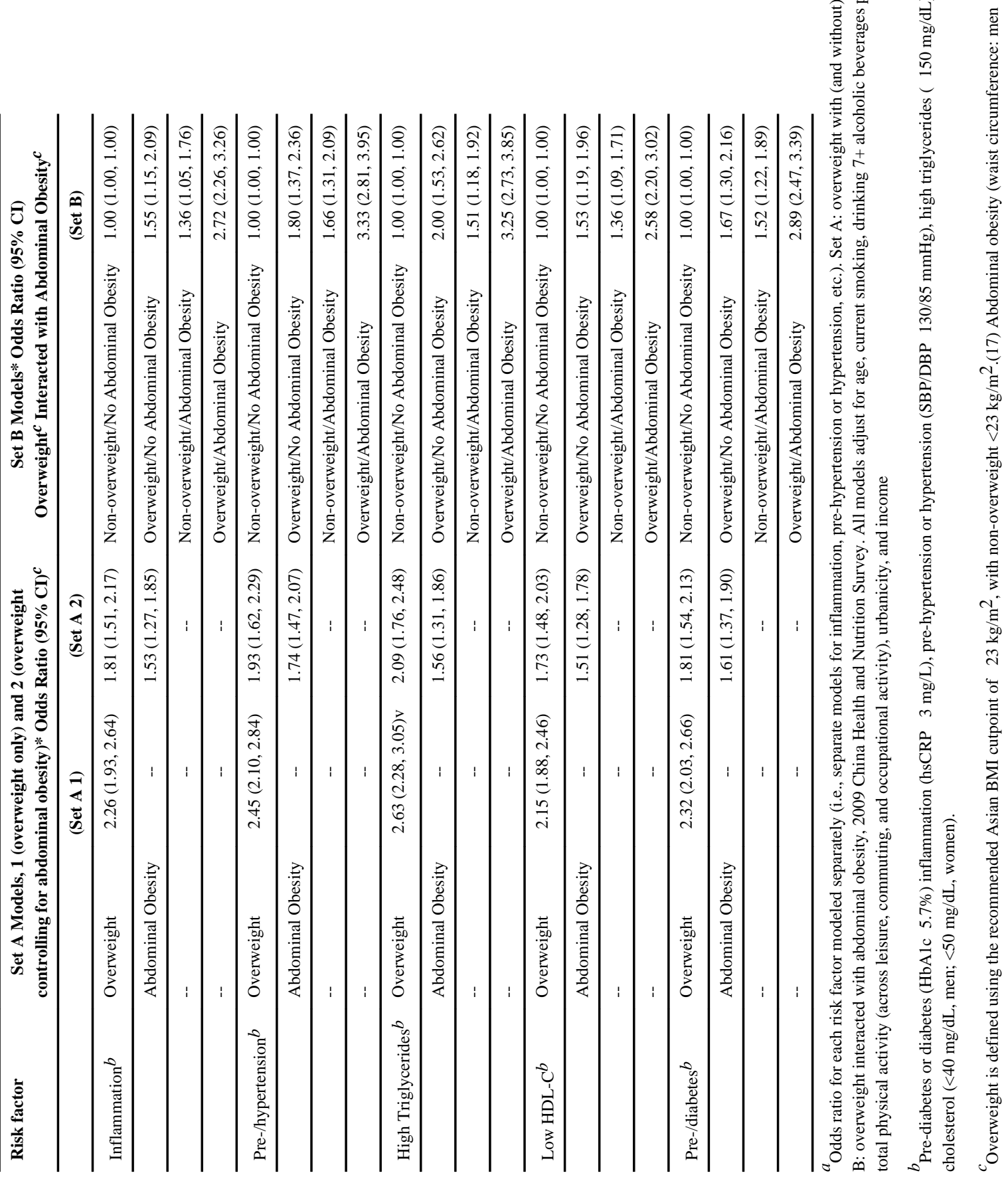




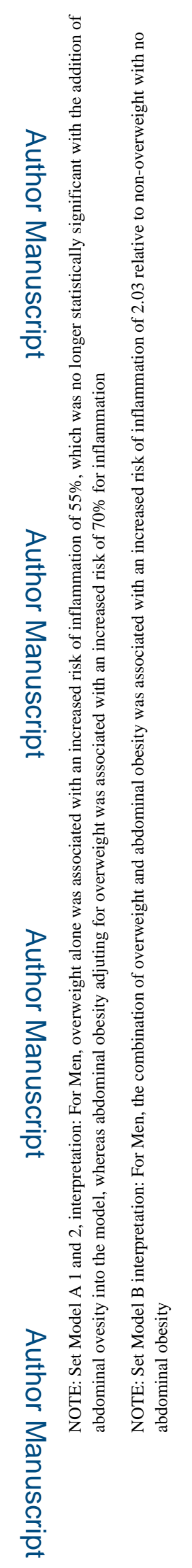

